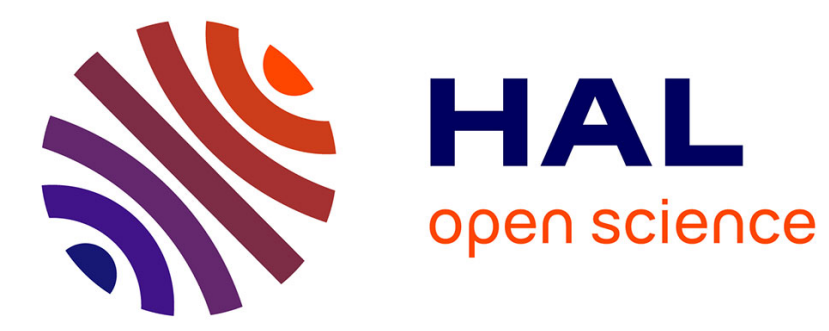

\title{
La réalisation des réseaux de desserte. Les schémas de desserte de voirie et d'équipement forestier dans les Pyrénées. L'exemple du département de l'Aude
}

\author{
M. Deblaize
}

\section{- To cite this version:}

M. Deblaize. La réalisation des réseaux de desserte. Les schémas de desserte de voirie et d'équipement forestier dans les Pyrénées. L'exemple du département de l'Aude. Revue forestière française, 1987, 39 (S), pp.97-102. 10.4267/2042/25857 . hal-03424708

\section{HAL Id: hal-03424708 \\ https://hal.science/hal-03424708}

Submitted on 10 Nov 2021

HAL is a multi-disciplinary open access archive for the deposit and dissemination of scientific research documents, whether they are published or not. The documents may come from teaching and research institutions in France or abroad, or from public or private research centers.
L'archive ouverte pluridisciplinaire HAL, est destinée au dépôt et à la diffusion de documents scientifiques de niveau recherche, publiés ou non, émanant des établissements d'enseignement et de recherche français ou étrangers, des laboratoires publics ou privés. 


\title{
LA RÉALISATION DES RÉSEAUX DE DESSERTE LES SCHÉMAS DE DESSERTE DE VOIRIE ET D'ÉQUIPEMENT FORESTIER DANS LES PYRÉNÉES
}

\section{L'exemple du département de l'Aude}

\author{
M. DEBLAIZE
}

\begin{abstract}
"Faute de dégagement, certaines forêts sont inexploitables ou à des prix décourageant toute gestion sérieuse. En outre certaines voies publiques sont peu accessibles aux véhicules lourds grumiers. Des investissements importants sont à consentir mais encore faut-il veiller, d'une part à ne pas assimiler route forestière et route touristique..., d'autre part à ne pas investir sans avoir réuni toutes les autres conditions d'une bonne valorisation de la forêt ".
\end{abstract}

C'est en ces termes que le Schéma d'Orientation des Pyrénées abordait, en 1977, le problème de la desserte des forêts et, d'une façon plus générale, celui de la mise en valeur de la forêt pyrénéenne dont il avait fait l'une de ses priorités.

Ce problème n'était d'ailleurs pas spécifique au Massif pyrénéen; les groupes "bois de trituration "l'avaient déjà abordé dans leurs régions; de même, le Service des Forêts d'alors, notamment le Fonds forestier national, s'y était intéressé.

Néanmoins, nous nous appuierons sur cet exemple des Pyrénées, et plus particulièrement sur celui du département de l'Aude, pour traiter des réseaux et des schémas de desserte.

\section{L'HISTORIQUE ET LE POURQUOI DU CONCEPT “DESSERTE FORESTIËRE "}

Parmi les faiblesses de la filière-bois si souvent dénoncées, il en est une qui, dans certaines zones montagneuses, revêt une acuité particulière parce qu'elle entrave la sylviculture et la mobilisation des produits: l'insuffisance de l'équipement routier des forêts.

Ajoutée à d'autres facteurs tels que le morcellement et les particularités structurelles de la forêt privée, la modernisation des moyens d'exploitation, la baisse des cours du bois, l'augmentation des coûts de la main d'œuvre, elle induit une sous-exploitation des forêts.

Ainsi, pour le Massif pyrénéen où les forêts couvrent plus de 500000 hectares, la récolte n'atteint pas moitié de l'accroissement biologique et, en 1980 , on estimait à $880000 \mathrm{~m}^{3}$ le manque à rècolter annuellement. Les coûts d'exploitation sont le double de ceux de plaine alors que le prix du bois $y$ est deux fois moins cher.

Les produits de faible valeur marchande comme les produits d'éclaircie, de coupe de taillis ou même les bois de fortes dimensions, sont difficilement exploitables à cause de leurs coûts 


\section{DEBLAIZE}

d'exploitation. Pourtant, ces coupes sont importantes; que seront en l'an 2000 les forêts artificielles créées par le FFN, si on n'y réalise pas les éclaircies qui s'imposent ? De même, en ce qui concerne les beaux produits, le sous-équipement routier entraîne une moins-value sur pied qui tend à déséquilibrer l'économie forestière.

Parmi les mesures susceptibles de résoudre ces problèmes, il est apparu indéniable que l'équipement routier occupait une place centrale et qu'un effort sélectif mené dans ce domaine était le moyen d'obtenir des résultats rapides et de provoquer un effet d'entrainement.

Sur les bases de ce constat, sous la direction de la Délégation à l'Aménagement du Territoire et avec l'appui du Service des Forêts (Fonds forestier national) et des délégués de Massif, c'est l'avènement, en 1979, du Fonds interministériel de Développement et d'Aménagement rural (FIDAR) qui permit la concrétisation du programme " desserte forestière ".

Après une concertation interrégionale, le Comité interministériel de Développement et d'Aménagement rural a, le 21 février 1980, approuvé le principe d'un programme pluriannuel de desserte forestière à deux volets:

- un volet "schémas de desserte "pour la réalisation des études de schémas financées à parts égales par le FFN et le FIDAR. Avec la coordination et l'appui logistique des échelons régionaux de l'Administration forestière (SERFOB - CRPF Midi-Pyrénées), des chargés d'étude furent mis en place à raison d'un pour deux départements. Ces chargés d'études travaillaient sous le contrôle de groupes départementaux. Les ètudes ont été conduites avec des fortunes diverses pendant deux années avant d'être abandonnées en 1982 :

- parce que jugées trop lourdes à l'échelle de la chaîne pyrénéenne :

- par manque de conviction dans certains départements.

Dans le département de l'Aude malgré une année d'interruption, les études se poursuivent toujours grâce à des financements FIDAR et les schémas sont suivis de réalisations concrètes.

- un volet "réalisation et financement des projets " inclus dans les schémas, sur les bases d'un cofinancement FFN et FIDAR.

\section{LES OBJECTIFS DU PROGRAMME PLURIANNUEL DE DESSERTE}

L'objectif assigné au programme pluriannuel était triple:

- donner à la desserte des forêts l'impulsion nouvelle nécessaire à l'accroissement souhaité de la mobilisation de la ressource;

- créer un outil de programmation et d'aménagement assurant une cohérence des équipements entre eux mais aussi avec les textes en vigueur (par exemple la Directive Montagne du 22 novembre 1977 qui limitait au strict nécessaire les créations de routes), avec les autres usages de la montagne (ski, tourisme, pastoralisme...);

- participer au développement économique local (objectif FIDAR).

De fait, les schemas de desserte, et leurs réalisations:

- en facilitant l'accès de la forêt par véhicule aux bûcherons et exploitants :

- en réduisant les distances de débusquage, de halage, de câblage, de lançage;

- en permettant une intensification de l'emploi des tracteurs forestiers;

- en permettant aux camions grumiers l'accès au cœur de la forêt ;

doivent permettre une meilleure mobilisation de la ressource par:

- la réduction des coûts d'exploitation, 
- l'ouverture vers une mécanisation poussée,

- la répercussion de ces économies sur le prix des bois sur pied qui devient ainsi plus incitatif pour le propriétaire,

- la réalisation des coupes invendables sans cela (éclaircies).

Par ailleurs, grâce aux regroupements [Association syndicale autorisée (ASA)] qu'ils induisent, ils doivent permettre d'intensifier la gestion des forêts. Enfin, ils rendent possible l'ensemble des travaux d'entretien qui conditionnent l'état futur de la forêt.

Il va sans dire que cette action d'amont devrait permettre aux industries de transformation du bois de disposer, le moment venu, de l'essentiel d'une ressource adaptée à leurs exigences malgré l'accroissement important qu'elle va connaître dans un proche avenir, grâce aux reboisements du FFN.

\section{LES SCHÉMAS DE DESSERTE}

Pour répondre à l'ensemble des problèmes posés, un schéma de desserte forestière doit être un document de référence, d'aménagement et de programmation, simple, précis et concis. II doit notamment comprendre :

- sur une aire géographique limitée à un massif ou une vallée, le tracé de l'ensemble des voies forestières primaires, cohèrent avec les autres voiries (départementale, communale, touristique...), des voies forestières secondaires (pistes) et bien sûr des voies existantes ;

- une carte des peuplements avec l'estimation des surfaces, des volumes à mobiliser par nature de peuplements et de propriétaires;

- une étude économique devant faire ressortir l'intérêt et la rentabilité de l'opération (coût de premier établissement en égard aux plus-values attendues sur les bois, les travaux, les autres activités...);

- un programme de mise en valeur qui accompagnera chaque projet (reboisement, conversion, commercialisation...);

- une étude d'impact liée à l'environnement, l'emploi, l'activité aval, au contexte social.

A l'échelle du Massif pyrénéen, 13 schémas ont été réalisés; ils prévoient une longueur de voies de desserte de plus de 600 kilomètres et un volume de bois supplémentaire annuel de l'ordre de $65000 \mathrm{~m}^{3}$.

Dans le département de l'Aude, 3 schémas ont déjà été réalisés sur les Pyrénées et 1 sur le Massif Central (Montagne Noire).

\section{LES SCHÉMAS PYRÉNÉENS DU DÉPARTEMENT DE L'AUDE}

\section{Le schéma du Massif de Madres}

La zone de Madres constitue un massif d'une surface de 11300 ha dont 4300 ha de forêt privée délimitée par les vallées de l'Aude et de la Boulzane et les limites départementales avec l'Ariège et les Pyrénées-Orientales. Elle constitue l'étage supérieur de la sapinière audoise. Le schéma a déterminé 13 projets urgents totalisant une longueur de route d'environ $80 \mathrm{~km}$. Fin 1986 , ce sont 8 projets, d'une longueur de $35 \mathrm{~km}$ et d'un montant de $5000000 \mathrm{~F}$ qui ont vu le jour avec un cofinancement FFN-FIDAR pour 7 d'entre eux. 


\section{Le schéma de la vallée du Rebenty}

Cette région constitue un massif d'une surface de 10000 ha de forêts, dont 4000 ha de forêts privées, occupant principalement le versant Nord de la vallée du Rebenty, affluent de l'Aude.

Le schéma a déterminé 8 projets urgents totalisant une longueur de $45 \mathrm{~km}$. En 1986, ce sont 3 projets, d'une longueur de $21 \mathrm{~km}$ et d'un montant de $5600000 \mathrm{~F}$, qui ont vu le jour avec un cofinancement FFN-FIDAR.

\section{Le schéma de la région de Chalabre}

Cette zone constitue un massif de piémont, avec néanmoins des conditions très difficiles de relief, d'une surface de 11000 hectares dont seulement 900 ha de forêts publiques, situé au Sud-Ouest du département, au contact de l'Ariège. La forêt est une forêt essentiellement paysanne, morcelée, qui nécessitera le recours à des structures collectives.

Comme dans toutes les zones montagneuses de piemont, la sortie par les vallées y est impossible à cause des points noirs habituels (ponts, villages...); il est nécessaire de réaliser la sortie par les crêtes.

Le schéma a déterminé 27 projets totalisant une longueur de $150 \mathrm{~km}$.

Fin 1986, ce sont 4 projets individuels privés de $16 \mathrm{~km}$ et d'un montant de $2400000 \mathrm{~F}$ qui ont été proposés pour un financement FFN uniquement, le FIDAR n'intervenant pas pour des projets privès $(P \mid M)$.

\section{Perspectives d'avenir}

Dans le département de l'Aude, les schémas de desserte étudiés ont donc été suivis de réalisations concrètes, surtout pour les deux premiers schémas intéressant des zones sans problèmes fonciers importants, ne nécessitant pas la mise en place de structures collectives.

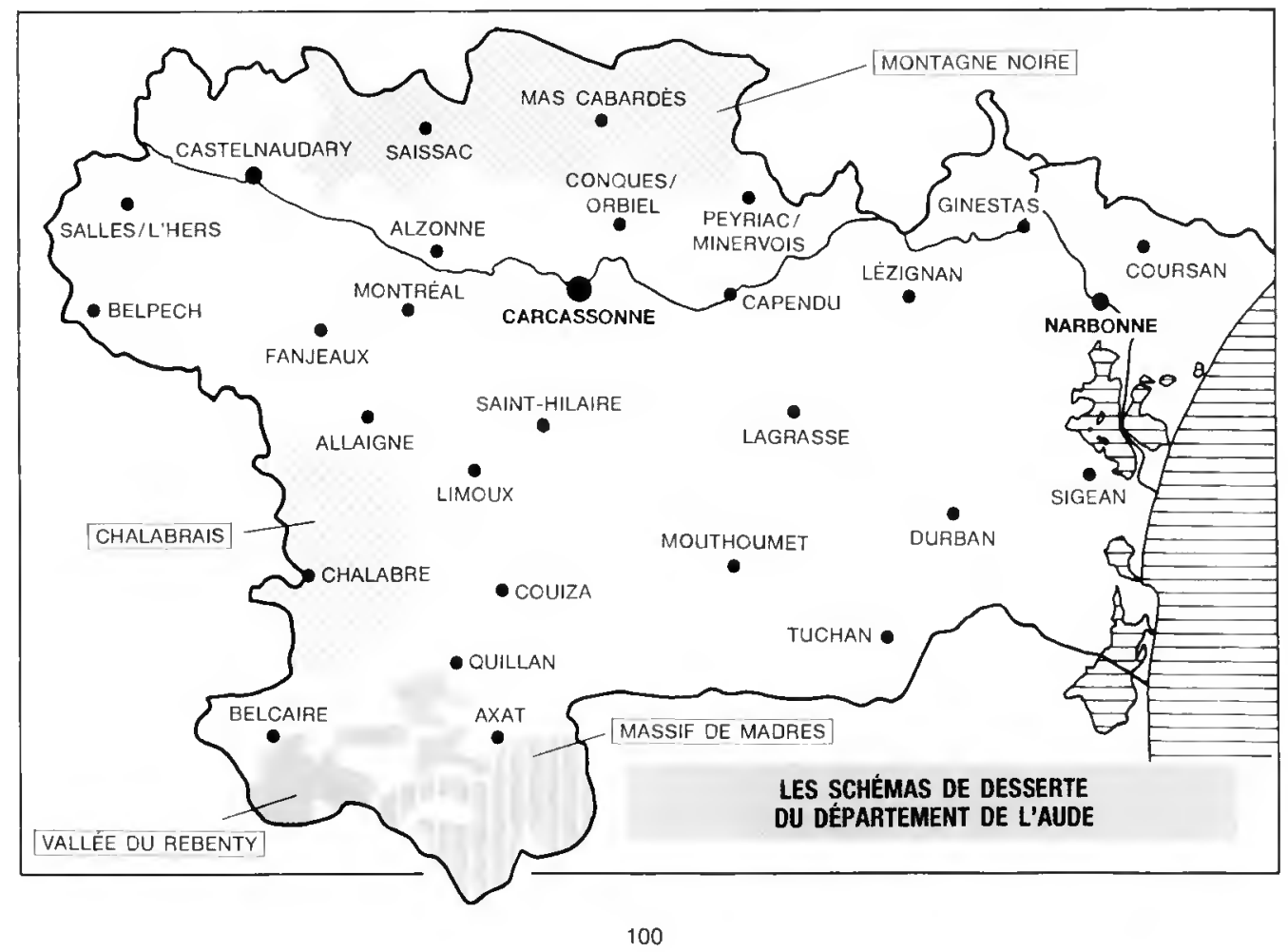




\section{L'équipement forestier}

Pour la troisième zone où des problèmes fonciers se posent et où des structures collectives seront nécessaires, les réalisations devront se faire à un rythme plus lent.

Les objectifs tels que définis précédemment ont èté atteints dans leur ensemble; les schémas sont notamment un outil intéressant de programmation.

\section{LES SCHÉMAS ET LA VOIRIE AVAL}

Nous avons indiqué plus haut qu'un schéma de desserte devait être cohérent avec les autres voiries existantes. Ainsi pour la voirie aval, des programmes pluriannuels de voirie publique de vidange des bois ont été élaborés en 1981. Ils devaient donner, au niveau des départements, la liste des points noirs routiers faisant obstacle à une bonne sortie des bois et l'évaluation des travaux de résorption. Ceux-ci devaient être financés sur le Fonds spécial d'Investissement routier (FSIR) grâce à des enveloppes spécifiques supplémentaires.

Si certains départements ont joué le jeu, d'autres comme le département de l'Aude, ne l'ont pas fait ; les enveloppes supplémentaires, noyées dans la masse du FSIR, ont été utilisées à d'autres fins. Seuls 3 points noirs, sur les 15 inventoriés, ont été résorbés fortuitement.

II faut regretter que de telles actions ne fassent pas l'objet d'une gestion spécitique.

\section{CRITIQUES}

L'ambition des schémas de desserte était en fait trop grande; c'est ce qui a conduit à l'abandon de 1982 au niveau de la chaine pyrénéenne.

Les causes de cet échec sont:

- la multiplicité des facteurs à prendre en compte (tourisme, TDF, pastoralisme, forêt, ski...);

- l'antagonisme trop fréquent de ces facteurs (élevage-forêt par exemple) ;

- la conception envisagée de ces schémas, certes séduisante mais beaucoup trop technocratique ;

- le dérapage des schémas du pratique vers l'économique avec des calculs d'échéanciers quelque peu utopistes (cf. l'évaluation des projets forestiers par l'OCDE en 1981), alors que l'aspect foncier, autrement important, est oublié.

En zone de forêts soumises, les schémas sont trop souvent le recueil des projets de l'ONF au détriment de la forêt privée.

En ce qui concerne le financement, le Fonds forestier national a toujours joué le jeu en acceptant de financer tous les projets présentés. Par contre le FIDAR a trop souvent agi comme un outil politique comme en témoignent:

- sa réticence à tinancer les premiers projets présentés en 1982 ;

- son refus de financer les projets privés;

- sa décision en 1984 de ne financer que des projets de communes adhérentes à un syndicat des communes, position qui a été élargie en 1986. 


\section{CONCLUSIONS}

Les objectifs des schémas de desserte, tels que définis plus haut, n'ont pas toujours été atteints au niveau de la chaîne pyrénéenne. Néanmoins, les schémas ont permis de mener une réflexion globale sur le problème de la desserte forestière tant au niveau de la chaine, qu'au niveau des départements et enfin des secteurs d'étude. Ils ont permis aussi une réflexion sur les problèmes fonciers en liaison avec la gestion forestière.

Enfin, ils jouent bien leur rôle d'outil de programmation cohérente pour l'Administration forestière.

M. DEBLAIZE

DIRECTION DÉPARTEMENTALE DE L'AGRICULTURE ET DE LA FORÊT

3, rue Trivalle

11000 CARCASSONNE 